\title{
$N$-磺基榺类化合物合成研究进展
}

\author{
郑莤茜刘云云* 万结平* \\ (江西师范大学化学化工学院 南昌 330022)
}

\begin{abstract}
摘要 脒类化合物因其独特的偕二氮结构, 在合成含氮化合物方面具有重要作用. 作为脒类化合物中独特的一类, $N-$ 磺基榺类化合物更是多种重要有机合成反应的关键中间体，在现代有机合成中占有举足轻重的位置. 基于这一背景, 对 $N$-磺基缕类化合物的合成进展进行了综述. 依据反应特点, 分别基于胺氧化酰化反应、炔-叠氮-胺三组分反应、烯 胺碳一碳键官能化、酰胺活化以及其它相关的反应等方面的研究进展进行介绍, 以期为相关领域的研究工作提供指导作 用.
\end{abstract}

关键词 $N$-磺基脒; 合成; 胺氧化; 酰胺活化; 三组分; 烯胺反应

\section{Advances in the Synthesis of $\mathrm{N}$-Sulfonyl Amidines}

\author{
Zheng, Xixi Liu, Yunyun* Wan, Jie-Ping* \\ (College of Chemistry and Chemical Engineering, Jiangxi Normal University, Nanchang 330022)
}

\begin{abstract}
Due to the special gem-nitrogen structure, amidines are important compounds in the synthesis of nitrogen contaning products. As a class of featured amidines, the $N$-sulfonyl amidines are key intermediates in a number of pivatol organic syntheses, and thus ocuppy significant position in modern organic synthesis. In this context, the research advances on the synthesis of $N$-sulfonyl amidines are herein reviewed. Based on the key reaction features, the introduction covers the synthetic methods of enamination by tertiary enamine oxidation, amide activation, akyne-azide-amine three-component reaction, enamine carbon-carbon bond functionalization and other related reactions. It is expected that this review will provide guidelines for the reasearch work in related areas.
\end{abstract}

Keywords $N$-sulfonyl amidine; synthesis; amine oxidation; amide activation; three-component; enamine reaction

脒类化合物含有独特的 $\mathrm{N}-\mathrm{C}=\mathrm{N}$ 结构, 该结构存 在于许多天然产物和生物活性小分子中，也是重要的生 物药物功能基团 ${ }^{[1]}$. 其中, $N$-磺基脒是椫类化合物中较 为特别的一类, 其结构大多稳定, 在生物药物分子设计 和有机合成化学上都有广泛的应用. 例如, $N$-磺基椫不 仅是合成杂环化合物, 尤其是嘧啶类杂环化合物的关键 砌块, 同时还可作为鳌合剂作用于过渡金属, 对某些蛋 白酶具有抑制活性等丰富的生物功能 ${ }^{[2]}$. 其中, 脒结构 对炭瘨中一种致命的锌离子金属蛋白酶的抑制功能正 是基于这一原理 ${ }^{[3]}$. 此外, 磺基椫类化合物还被发现具 有重要的吸收抑制 ${ }^{[4]}$ 、抗癌 ${ }^{[5]}$ 及抗增殖 ${ }^{[6]}$ 等药物活性 ${ }^{[7]}$, 可用于药物先导化合物的合成研究. 基于 $N$-磺基椫类 化合物在多个领域的重要应用前景, 对于这类化合物的 合成研究也成为有机合成研究领域的重要课题 ${ }^{[8]}$. 值得
注意的是, 脒类化合物极性较大, 在合成时应特别注意 分析.

在过去的十多年时间，对于 $N$-磺基脒的化合物的 合成研究取得了令人瞩目的进展. 目前已经有许多可高 效合成 $N$-磺基椫类化合物的方法. 然而, 到目前为止, 尚没有对该研究领域研究进展的综述性工作. 因此, 有 必要对这一领域的研究进展进行系统总结, 为朕类化合 物的合成以及相关应用提供一些参考. 基于已有相关文 献报道方法的特征, 本文从三级胺氧化酰胺化促进的合 成、酰胺活化、炔-叠氮-胺三组分反应合成、基于烯胺 碳一碳键的官能化合成以及其它相关合成方法几方面, 对 $N$-磺基脒类化合物的合成研究进行系统的梳理和总 结, 展示该研究领域的进展和现状, 同时梳理出存在的 挑战以及可能努力的方向.

\footnotetext{
* Corresponding authors. E-mail: chemliuyunyun@jxnu.edu.cn; wanjieping@jxnu.edu.cn Received March 8, 2020; revised April 1, 2020; published online April 11, 2020.

Project supported by the National Natural Science Foundation of China (No. 21861019). 国家自然科学基金(No. 21861019)资助项目.
} 


\section{1 胺氧化烯胺化反应合成 $N$-磺基膦}

通过三级胺原位活化形成烯胺中间体, 再和磺酰叠 氮通过 $[3+2]$ 环加成形成 1,2,3-三唑啉中间体，该中间 体在适合条件下，通过杂环的分解，脱除重氮后，可以 得到 $N$-磺基脒类产物. 这类反应可以在不同条件进行, 在磺基榺合成方面具有重要应用. 如 Scheme 1 所示, 这 类合成方法对底物有一定要求, 即需要用到含有乙基结 构的三级胺作为前体. 同时, 胺结构中如有超过一个不 等同的乙基, 在反应过程中则会生成不同位点氧化导致 的副产物.

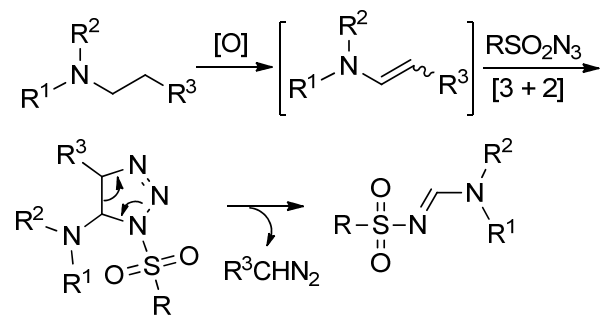

图式 1 基于三级胺氧化烯胺化合成 $N$-磺基脒 Scheme $1 \mathrm{~N}$-Sulfonyl amidine synthesis by oxidative tertiary amine enamination

2008 年，李小年等 ${ }^{[9]}$ 报道了通过偶氮二甲酸二乙酯 (DEAD)促进叔胺和磺酰叠氮反应合成 $N$-磺基脒类化合 物的方法. 该工作中, DEAD 作为氢受体氧化叔胺得到 烯胺中间体，随后该中间体和磺酰叠氮发生 1,3-偶极环 加成，生成三唑啉中间体. 该中间体在反应条件下发生 分解, 释放出一分子重氮甲烷, 从而得到 $N$-磺基榺产物 (Scheme 2).

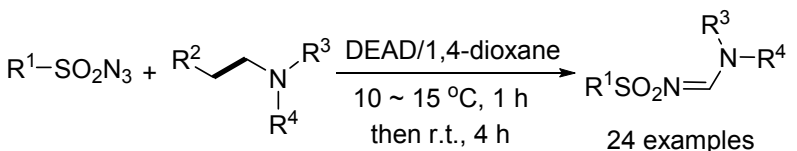

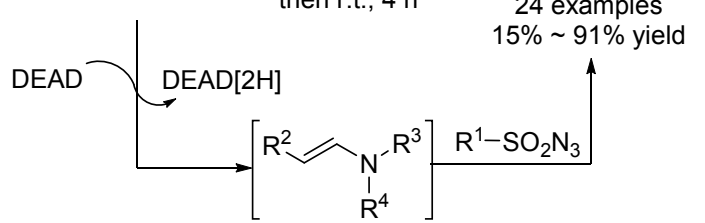

图式 2 DEAD 促进三级胺氧化烯胺化合成 $N$-磺基脒 Scheme2 DEAD promoted $N$-sulfonyl amidine synthesis via tertiary amine oxidation

之后, 该课题组 ${ }^{[10]}$ 又报道了 $\mathrm{CuCl} / \mathrm{CCl}_{4}$ 体系催化三 级胺脱氢烯胺化和磺酰叠氮合成磺基脒的反应. 而且反 应在无催化剂, 室温条件下也可以进行，但反应时间太 长. 添加 $\mathrm{CuCl}$ 可使产率提高并大大缩短反应时间. 研 究还揭示 $\mathrm{CCl}_{4}$ 不仅作为溶剂，还参与到了该反应中，帮 助形成烯胺中间体. 更换叔胺底物得到的 $N$-磺基脒产 物结构主要取决于叔胺上的取代基. 部分叔胺由于存在
不同可参与反应的氧化位点, 得到两种 $N$-磺基脒产物 (Scheme 3).

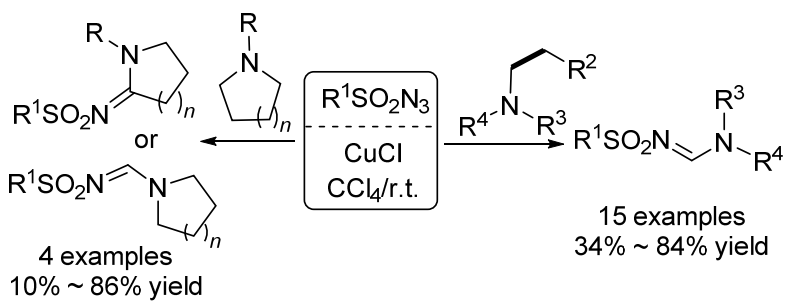

图式 $3 \mathrm{CuCl}$ 催化三级胺氧化烯胺化合成 $N$-磺基咪唑 Scheme $3 \mathrm{CuCl}$-catalyzed synthesis of $\mathrm{N}$-sulfonyl amidines via oxidative tertiary amine enamination

何玲等 ${ }^{[11]}$ 也报道过用 $\mathrm{CuCl}$ 作为催化剂催化三级胺 与磺酰叠氮得到 $N$-磺基脒产物的反应，较上述文献方 法不同的是该研究小组使用了乙腈作为溶剂并回流，并 以双磺基氮烯作为氢受体来促进三级胺的氧化. 磺酰叠 氮方面，只有芳基磺酰叠氮可参与反应，烷基磺酰叠氮 不适用(Eq. 1).

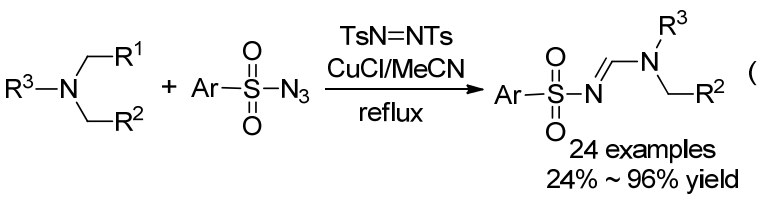

毕文柱等 ${ }^{[12]}$ 报道了磺酰氯、叠氮化钠和胺在空气中 通过溴化铜催化合成 $N$-磺基脒的方法. 该方法的优势 在于磺酰叠氮底物不需要预先合成, 通过磺酰氯和叠氮 化钠原位反应得到. 反应体系中采用空气中的氧作为氧 化剂，在原子经济性方面也具有优势(Eq. 2).

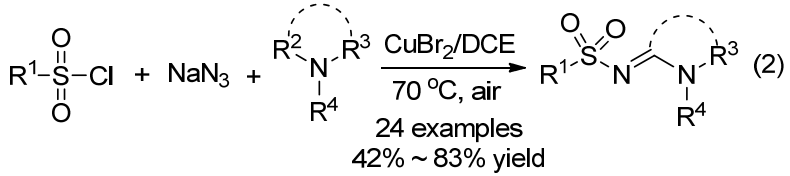

同样采用空气氧化，汪志勇等 ${ }^{[13]}$ 使用廉价的 $\mathrm{FeCl}_{3}$ 为促进剂，在二氯甲烷介质中，室温下实现了叔胺与磺 酰叠氮的反应，合成了 $N$-磺基榺类化合物. 值得一提的 是采用 $\mathrm{FeCl}_{3} \cdot 6 \mathrm{H}_{2} \mathrm{O}$ 作为催化剂则无法取得 $\mathrm{FeCl}_{3}$ 一样的 效果. 此外, 该条件下以环叔胺为底物得到的产物主要 以环碳上的脒产物为主, 证实 $\mathrm{FeCl}_{3}$ 对环上的 $\alpha-\mathrm{C}-\mathrm{H}$ 键 有更好的区域选择性(Scheme 4).

乌斯曼等 ${ }^{[14 a]}$ 在 1,2 -二氯乙烷 $(\mathrm{DCE})$ 中 $80{ }^{\circ} \mathrm{C}$ 加热, 采用 T-HYDRO (TBHP 摩尔分数为 $70 \%$ 的水溶液)促使 磺酰叠氮和叔胺或仲胺反应生成 $N$-磺基脒产物. T-HYDRO 在该反应中作为氧化剂，通过单电子转移实 现叔胺氧化得到烯胺中间体，再经过通用的转化途径生 成 $N$-磺基脒(Eq. 3). 夏吾畑等 ${ }^{[14 \mathrm{~b}]}$ 采用硝基苊作为光催 


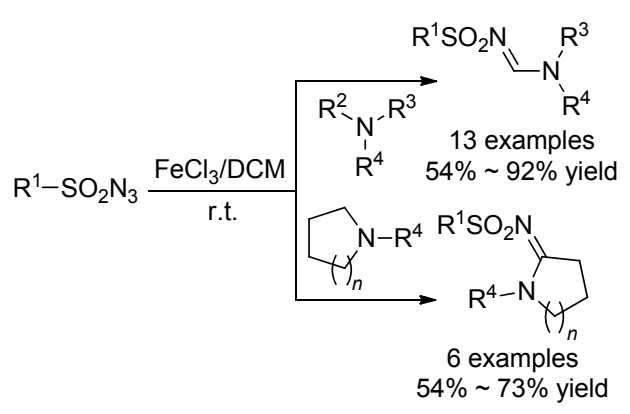

图式 4 三氯化铁催化胺和磺酰叠氮反应合成 $N$-磺基缕

Scheme $4 \quad \mathrm{FeCl}_{3}$-catalyzed synthesis of $N$-sulfonyl amidines via the reactions of amines and sulfonyl azides

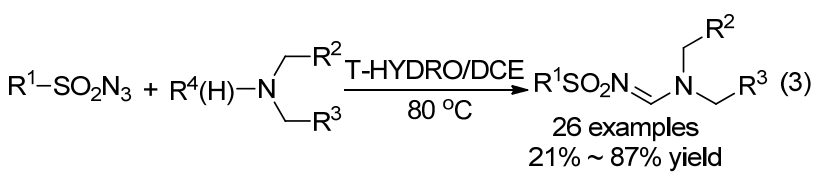

化剂, 实现了三乙胺和磺酰叠氮在光催化下合成 $N$-磺 基醚的类似反应.

Shahrisa 等 ${ }^{[15]}$ 使用磁性纤维素负载的 $N$-杂环卡宾铜配合物(NHC-Cu@MCs)多相纳米催化剂催化二级胺、 磺酰叠氮和三乙胺三组分反应, 实现了 $N$-磺基榺的合 成. 该方法中使用的催化剂可回收和重复使用, 且通过 直接采用二级胺在三乙胺基础上反应，提高了产物氨基 结构的多样性(Eq. 4).

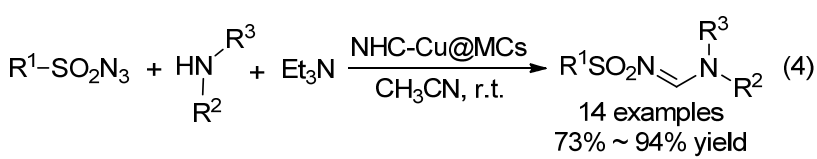

除了常规的热氧化方法, 光催化氧化反应也被证实 是实现该类 $N$-磺基脒类化合物的合成的有效方法. 曾 伟等 ${ }^{[16]}$ 用 Eosin $Y$ 为光催化剂, 在室温 $30 \mathrm{~W}$ 的蓝色光的 照射条件下, 建立了芳基磺酰叠氮与三级胺交叉反应合 成 $N$-磺基脒的方法. 反应首先是光催化剂在蓝光照射 下被诱导成激发态, 经历单电子转移、氧气氧化模式下 的胺氧化过程, 所得烯胺中间体和磺酰叠氮反应顺利得 到目标产物(Scheme 5).

潘英明等 ${ }^{[17]}$ 以 $\mathrm{Mes}-\mathrm{Acr}-\mathrm{Ph}^{+} \mathrm{BF}_{4}{ }^{-}$为光催化剂, 在二 氯甲烷中用 $12 \mathrm{~W}$ 的蓝色 LED 光照射, 室温条件下也可 实现这类基于磺酰叠氮和叔胺反应合成 $N$-磺基脒(Eq. 5). 有趣的是, 除了光催化氧化, 电催化方法也可促进 这一合成反应. 汪志勇课题组 ${ }^{[18]}$ 组用基于碳电极阳极 氧化策略, 室温下以乙腈为介质, $n-\mathrm{Bu}_{4} \mathrm{NPF}_{6}$ 为电解质, 可高效实现磺酰叠氮与叔胺经历胺氧化烯胺化以及后 续的串联环加成一分解反应，合成 $N$-磺基脒类化合物. 该方法除可用于叔胺的反应，还可顺利实现仲胺、伯胺 参与的反应，大大提高了脒产物结构的多样性.

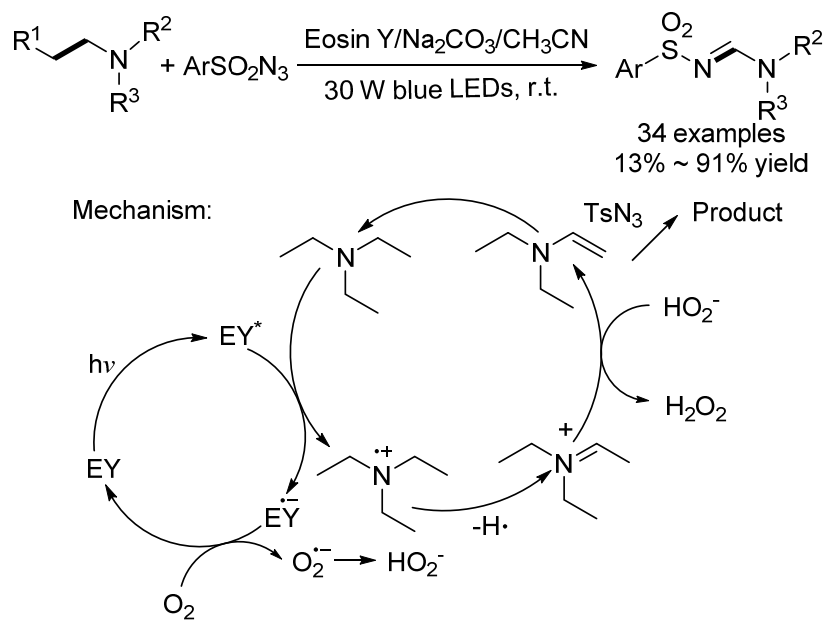

图式 5 Eosin $Y$ 光催化反应合成 $N$-磺基榺

Scheme 5 Eosin Y-based photocatalytic synthesis of $N$-sulfonyl amidines

(Scheme 6).

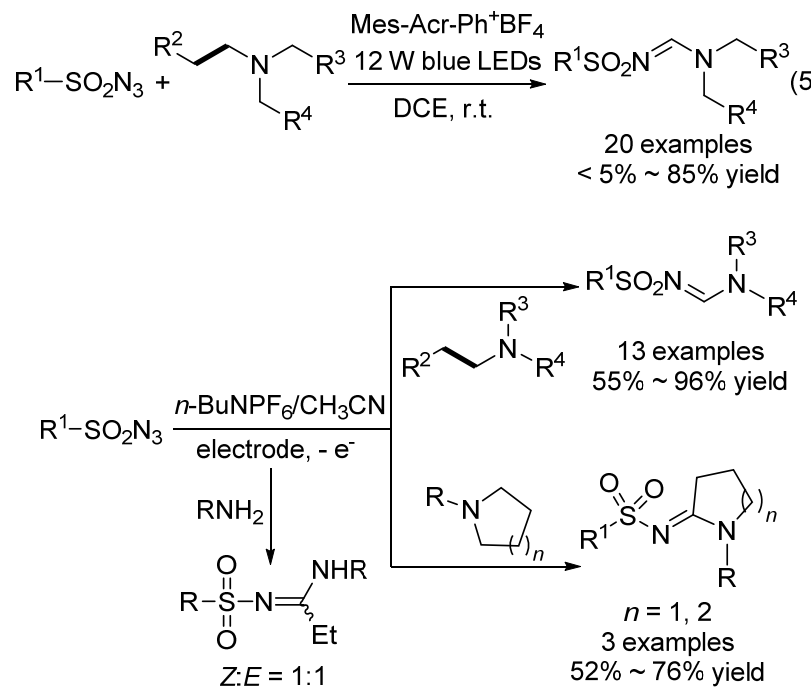

图式 6 电催化反应合成 $N$-磺基脒

Scheme 6 Synthesis of $N$-sulfamidine by electrocatalytic reaction

除了新催化氧化条件方面的研究, 底物结构的调整 也可提供新的合成方法. 周岭等 ${ }^{[19]}$ 报道了 $N$-甲基胺和 $N, N$-二溴磺酰胺或磺酰胺直接反应合成 $N$-磺基脒的方 法. 在 $N$-溴代丁二酰亚胺(NBS)和 1,5-二氮杂二环 [5.4.0]十一烯-5 (DBU)共同作用下，室温反应可得到 $N$ 磺基脒. 该反应中, $\mathrm{TsNBr}_{2}$ 既提供 $\mathrm{TsN}$-这部分的基团构 建产物，同时作为氧化剂氧化三乙胺产生烯胺中间体 (Scheme 7).

\section{2 基于烯胺碳-碳键的官能化合成 $N$-磺基畨}

在胺氧化合成 $N$-磺基脒的方法中，烯胺的生成是 整个反应过程的关键步骤. 因此, 通过稳定的烯胺直接 


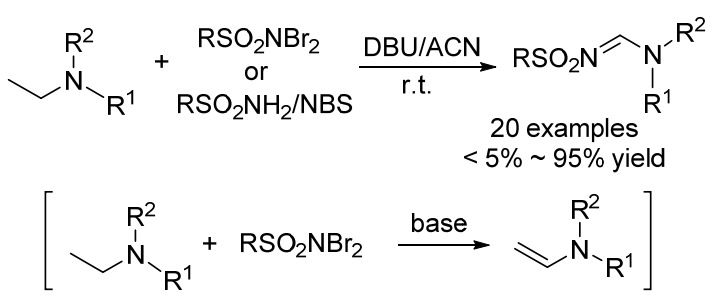

图式 $7 \mathrm{~N}, \mathrm{~N}$-二溴磺酰胺参与的反应合成 $N$-磺基榺

Scheme 7 Reactions of $N, N$-dibromosulfonamides for the synthesis of $N$-sulfonyl amidines

和磺酰叠氮反应无疑可以成为替代的合成方法. 因为烯 胺结构在底物中已经存在, 在相应的合成反应中就无需 再使用氧化剂. 此外, 双键反应位点的预先置入还可避 免在后续反应中在其它位点上反应生成副产物, 故这类 合成方法具有独特优势. Fusco 等 ${ }^{[20]}$ 较早报道了烯胺与 磺酰叠氮反应生成 $N$-磺基椫的结果, 以不同的烯胺和 对甲苯磺酰叠氮反应生成 3 个对应 $N$-磺基畨(Scheme 8).

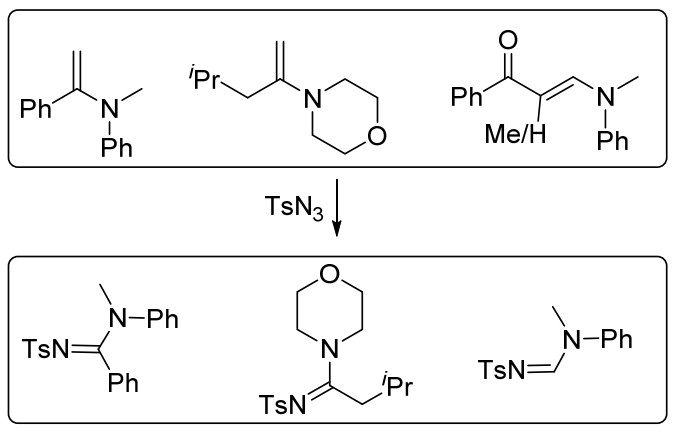

图式 8 烯胺和磺酰叠氮反应合成 $N$-磺基畨

Scheme 8 Reactions of enamines and tosyl azide for the synthesis of $N$-sulfonyl amidines

陈保华小组 ${ }^{[21]}$ 对类似合成方法进行了系统研究, 报道了在三乙胺促进条件下 $N, N$-二取代-2-苯基烯胺和 磺酰叠氮经过 1,3-偶极环加成及分解生成目标产物 $N$ 磺基脒的方法. 该方法反应时间短，在室温下搅拌 20 min 即可得到中等到优秀的产率, 并展示了末端烯胺更 广泛的底物范围(Eq. 6).

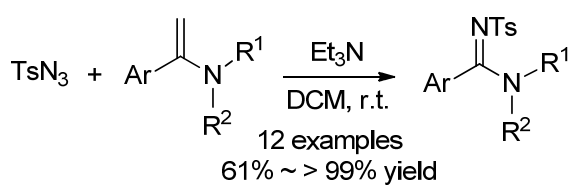

Bakulev 等 ${ }^{[22]}$ 通过进一步研究, 发展了采用环酮和 二级胺作为起始原料直接和磺酰叠氮反应的合成方法. 通过环酮和胺缩合原位生成烯胺中间体, 该中间体和磺 酰叠氮经历环加成以及分解反应即得到目标产物. 反应 以甲醇为介质在 $50{ }^{\circ} \mathrm{C}$ 加热即可实现. 除了磺酰叠氮, 该方法也适用于缺电子芳基官能化的叠氮底物的反应 (Scheme 9).

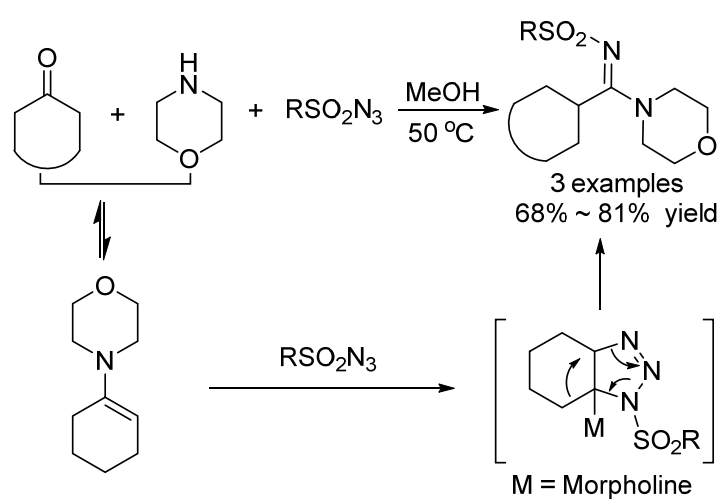

图式 9 环酮、胺和叠氮三组分反应合成 $N$-磺基脒 Scheme 9 Three-component reactions of cyclic ketone, amine and azide for the synthesis of $\mathrm{N}$-sulfonyl amidines

最近, 万结平小组 ${ }^{[23]}$ 报道了烯胺酯和磺酰叠氮反 应合成 $N$-磺基羘的绿色方法. 通过在烯胺结构上引入 酯基，即采用乙基烯胺酯和磺酰叠氮反应，在无需任何 催化剂、氧化剂或者添加物条件下，纯水作反应介质可 实现二者之间的反应生成 $N$-磺基缕. 反应底物适用范 围广, 大部分的乙基烯胺酯具有良好的反应效果. 平行 实验结果证实烯胺酯的存在，尤其是乙基酯的存在对反 应效果具有至关重要的作用(Scheme 10).

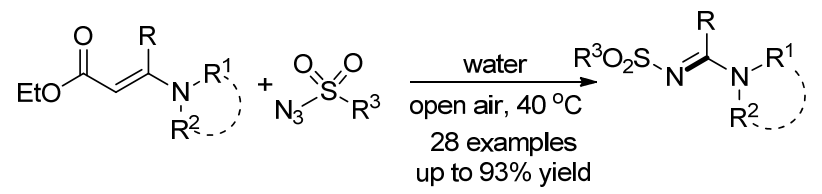

Mechanism:

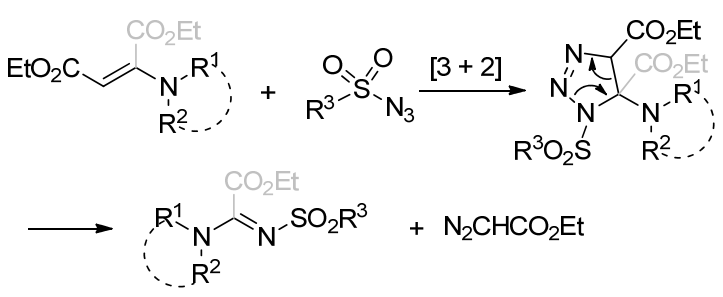

图式 10 烯胺酯和磺酰叠氮水相反应合成 $N$-磺基畨 Scheme 10 Aqueous reactions of enaminoesters and sulfonyl azides for $N$-sulfonyl amidines synthesis

有趣的是，在基于烯胺合成 $N$-磺基脒类化合物的 研究中, 绝大部分方法的适用性集中于 $N, N$-双取代结构 烯胺或烯胺中间体的反应，因而这类方法通常只适用于 相应的 $N, N$-双取代的磺基媨合成. 通过烯胺反应合成其 它类型氨基如 $\mathrm{NH}_{2}$ 官能化的磺基粗一直是该领域的一 个挑战. 最近, 万结平等 ${ }^{[24]}$ 通过采用 $\mathrm{NH}_{2}$ 结构的烯胺酮 为底物和磺酰叠氮在 $N, N$-二甲基甲酰胺(DMF)介质中 反应，在 DBU 促进下通过烯胺酮碳一碳键的断裂合成了 $\mathrm{NH}_{2}$-结构的 $\mathrm{N}$-磺基脒. 为这一挑战问题提供了新的解 决方案. 该工作还通过氮同位素标记的方法确证了产物 
中的 $\mathrm{NH}_{2}$ 来自烯胺酮底物, 反应可选择性成脒而不是三 氮唑的原因初步推测为碱添加物的存在抑制了通过释 放氨气生成三氮唑这一反应途径(Scheme 11).

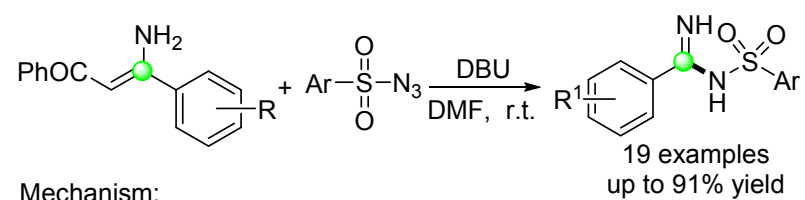

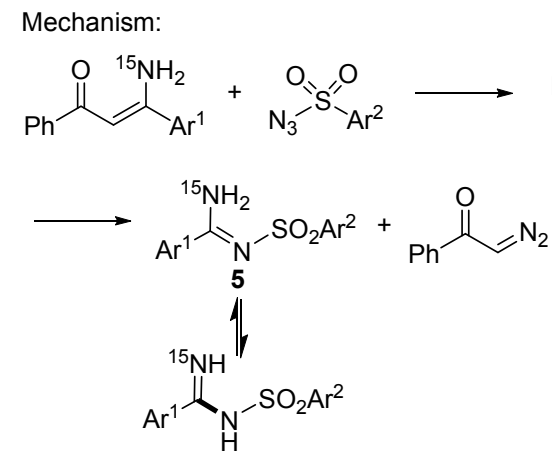
PhOC $\underbrace{\mathrm{Ar}_{\mathrm{N}}^{1}}_{\mathrm{N}=\mathrm{N}} \mathrm{NO}_{2} \mathrm{Ar}^{2}$<smiles>N[Y](N)(N)C[Al]c1c(C(=O)Oc2ccccc2)nnn1S(=O)O[Na]</smiles>

图式 11 烯胺酮和磺酰叠氮反应合成 $\mathrm{NH}_{2}$ 官能化的 $N$-磺基脒 Scheme 11 Reactions of enaminones and sulfonyl azides for the $\mathrm{NH}_{2}$-functionalized $\mathrm{N}$-sulfonyl amidines synthesis

\section{3 炔-叠氮-胺三组分反应合成 $N$-磺基膦}

炔、磺酰叠氮和胺的三组分反应也是合成 $N$-磺基榺 的一个重要方法. Chang 等 ${ }^{[25]}$ 最早报道了端炔、磺酰叠 氮和胺在亚酮催化剂下, 一锅法合成 $N$-磺基脒的方法. 该方法中一系列不同的胺类，包括含脂肪和芳基结构的 伯胺、仲胺均能参加反应. 通过同位素标记等控制实验, 作者提出了两种可能的反应历程: 一种是通过生成烯酮 亚胺中间体, 再和胺进行加成反应得到目标产物; 另一 种则是通过酰生成 1,2,3-三唑酮中间体，该中间体通过 脱氮还原生成 $N$-磺基脒(Scheme 12). 随后, 该课题组 ${ }^{[26]}$ 再次采用炔基官能化的胺作为底物和包括磺酰叠氮在 内的不同缺电子结构的叠氮在钉催化条件下, 实现了分 子内环化脒化反应, 建立了合成环胺结构脒类化合物的 方法(Scheme 12).

利用该合成方法, 以氯化铵作为胺源和端炔、2-溴 苯基磺酰叠氮反应, 得到的含 $\mathrm{NH}_{2}$ 结构的磺基脒产物可 在铜催化条件下进一步发生分子内碳一氮交叉偶联反

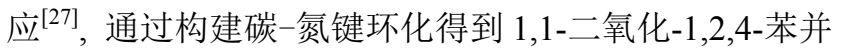
噻二嗪, 反应通过一锅法操作完成(Scheme 13), 且这类 杂环结构是重要的药效团 ${ }^{[28]}$.

Punniyamurthy 等 ${ }^{[29]}$ 将水杨醛作为额外底物引入这 类反应, 在 $\mathrm{CuI}$ 催化下合成了香豆素结构官能化的 $N$ 磺基榺类化合物. 该研究的思路是利用反应产生的脒结 构中的活泼亚甲基和水杨醛上的甲酰基脱水缩合引入 水杨醛结构, 然后再次利用水杨醛结构中的酚羟基和由 炔酯底物中引入的酯基发生交叉酯化反应, 通过环化

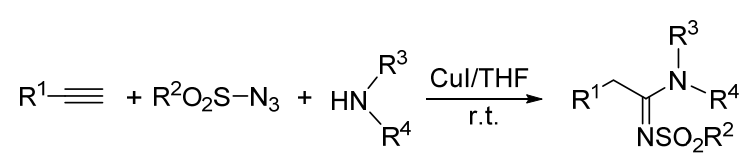
23 examples

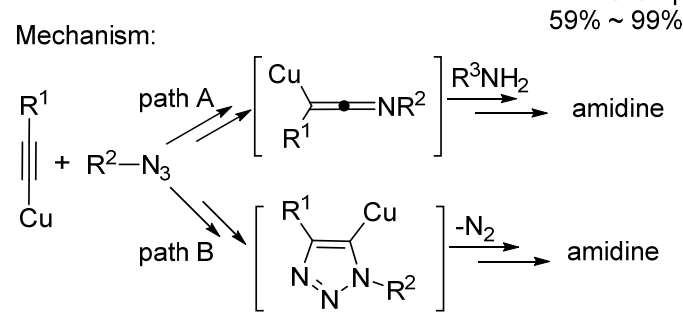

Intramolecular version

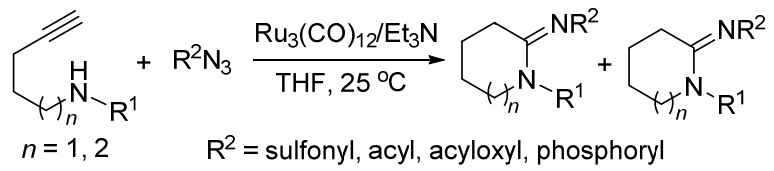

图式 12 端炔、胺和叠氮三组分反应合成脒

Scheme 12 Three-component reactions of terminal alkynes, amines and azides for amidines synthesis

$$
\begin{aligned}
& \mathrm{R}^{1}=\underset{\substack{\mathrm{Ar}=2-\mathrm{BrC}_{6} \mathrm{H}_{4} \\
\text { one-pot operation }}}{\mathrm{Ar}-\mathrm{SO}_{2} \mathrm{~N}_{3}+\mathrm{NHClH}} \frac{\mathrm{Cul} / \mathrm{Et}_{3} \mathrm{~N}}{\mathrm{DCM}, 25^{\circ} \mathrm{C}} \mathrm{R}^{1}
\end{aligned}
$$

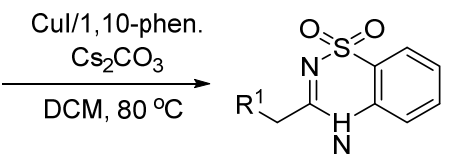

图式 13 基于 $N$-磺基榺中间体串联反应合成 1,1-二氧化-1,2, 4-苯并噻二嗪

Scheme 13 Synthesis of benzo[e][1,2,4]thiadiazine 1,1-dioxides via $N$-sulfonyl amidine intermediates

得到香豆素官能化的 $N$-磺基脒(Scheme 14).

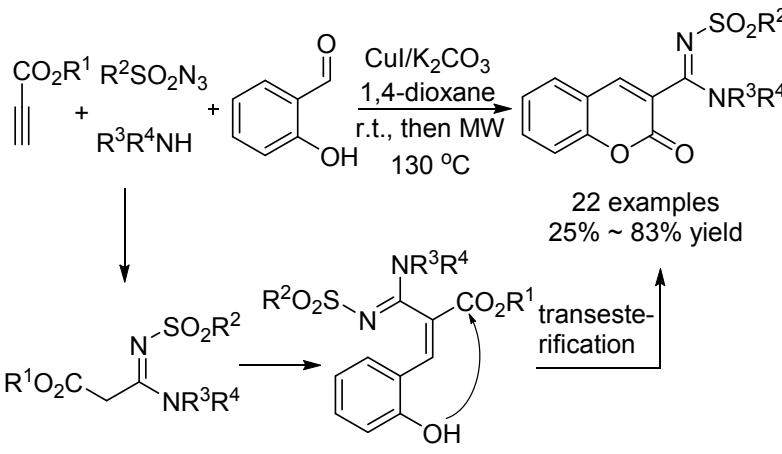

图式 14 四组分反应合成香豆素官能化的 $N$-磺基缕

Scheme 14 Four-component synthesis of coumarin functionalized $N$-sulfonyl amidines

在新催化条件的发展方面, Rashinkar 小组 ${ }^{[30]}$ 制备了 铜改性的钻沸石并将其作为催化剂, 实现了这类三组分 反应合成 $N$-磺基脒的方法. 反应在室温条件下进行，可 
用于伯胺和仲胺类底物的合成, 且催化剂可实现回收 (Scheme 15A). Kim 等 ${ }^{[31]}$ 使用通过金属有机物的非均相 铜试剂 $\mathrm{Cu}_{3}(\mathrm{BTC})_{2}$ 裂解所得 $\mathrm{Cu} @ \mathrm{C}$ 为催化剂催化端炔、 磺酰叠氮和仲胺三组分反应也可高效合成 $N$-磺基脒, 反应需在氮气保护条件下进行(Scheme 15B).

(A)

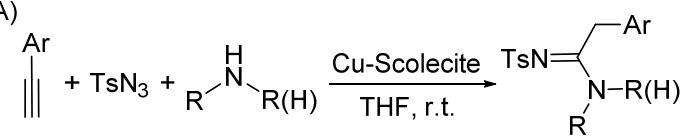

12 examples $61 \% \sim 90 \%$ yield

(B)

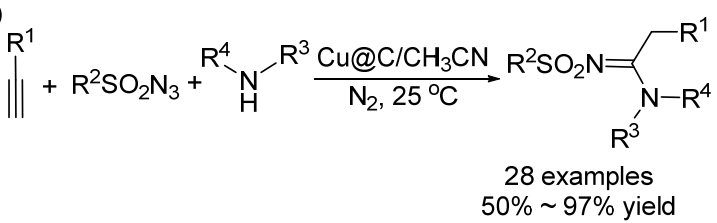

图式 15 不同类型铜催化剂催化炔、胺和磺酰叠氮三组分反 应合成 $N$-磺基榺

Scheme 15 Different copper catalysts for the three-component synthesis of $N$-sulfonyl amidines with alkyne, amine and azide

除了伯胺和仲胺, 三级胺也被证实可用作原料和 炔、磺酰叠氮反应合成磺酰叠氮类化合物. Yavari 等 ${ }^{[32]}$ 以 $\mathrm{CuI}$ 为催化剂, 在 $60{ }^{\circ} \mathrm{C}$ 加热条件下以端炔、磺酰叠 氮和三烷基胺反应合成了 $N, N$-双取代的磺基脒. 反应过 程中, 三烷基胺中的一个烷基通过和另一分子三级胺结 合, 以季胺盐的方式断裂碳-氮键得到目标产物(Scheme 16). 随后，商永嘉等 ${ }^{[33]}$ 采用偶氮异丁腈(AIBN)为引发 剂, 和 $\mathrm{CuI} / \mathrm{Et}_{3} \mathrm{~N}$ 组合成催化体系, 也实现了三级胺、炔 和磺酰叠氮构建 $N$-磺基脒的三组分反应.

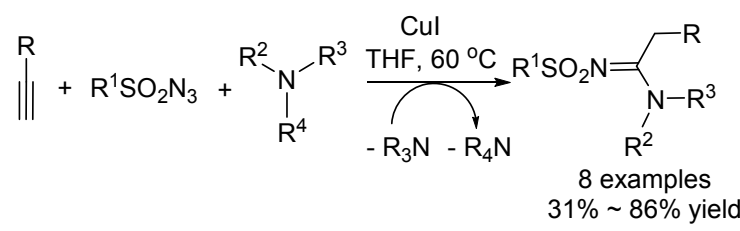

图式 16 三级胺、炔和磺酰叠氮三组分反应合成 $N$-磺基脒 Scheme 16 Synthesis of $N$-sulfonyl amidines with tertiary amines, alkynes sulfonyl azides

除了直接采用胺作为反应底物, 相关研究工作证实 了一些胺的衍生物或前体在适当条件下也可和炔以及 磺酰叠氮反应实现 $N$-磺基脒类化合物的合成. 张玉红 小组 ${ }^{[34]}$ 以 $\mathrm{CuBr}$ 为催化剂, dppe [1,2-双(二苯基膦)乙烷] 为配体, 在 $3 \AA$ 分子篎辅助下实现了端炔、磺酰叠氮以 及二烷基缩二甲酰胺类化合物的三组分反应，高效合成 了一系列烷氧基官能化的 $N$-磺基脒. 反应过程缩二甲 酰胺通过醇消除原位得到亚胺正离子, 该正离子和烯酮 亚胺中间体再原位反应, 经历异构化得到 $N$-磺基脒产 物(Scheme 17).

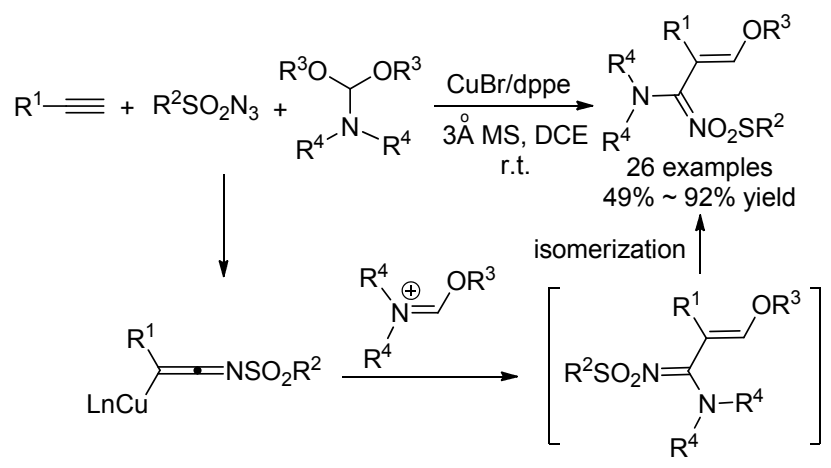

图式 17 缩二甲酰胺、炔和磺酰叠氮三组分反应合成 $N$-磺基 脒

Scheme 17 Synthesis of $N$-sulfonyl amidines with amide acetals, alkynes sulfonyl azides

\section{4 酰胺活化合成 $N$-磺基脒}

理论上说，酰胺同时具有氨基和羰基，可作为合成 脒类化合物前体. 因此发展通过酰胺活化合成脒的方法 具有重要意义. 然而, 由于酰胺本身的高度惰性, 这一 转化一直难以实现. 早期的转化都依赖于采用二甲亚砜 或三氯氧磷等烈性试剂将甲酰基转化为缩酰胺或活性 亚胺离子，后者再和磺酰胺缩合可得到磺基脒 ${ }^{[35]}$. 万小 兵等 ${ }^{[36]}$ 以磺酰胺和甲酰胺为原料, 在 $\mathrm{NaI}$ 和过氧化叔丁 醇(TBHP)体系中, $90{ }^{\circ} \mathrm{C}$ 加热实现了甲酰基和磺酰胺的 形式上缩合反应，得到了多种 $N$-磺基脒产物. 反应机理 可能是 $\mathrm{NaI}$ 使磺酰胺中的氨基去氢化活化, 形成活化的 亲核氨基再进攻甲酰胺，形成活性中间体后消除卤离子 和氧后就得到 $N$-磺基脒(Scheme 18).

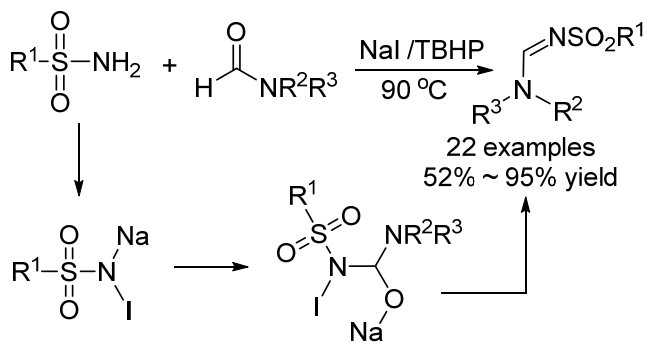

图式 $18 \mathrm{Na} / \mathrm{TBHP}$ 促进磺酰胺和酰胺反应合成 $N$-磺基脒 Scheme $18 \mathrm{NaI} / \mathrm{TBHP}$-promoted synthesis of $N$-sulfonyl amidines with amides and sulfonamides

在此基础上，该小组 ${ }^{[37}$ 还发展了通过金属卡宾中 间体活化甲酰胺的合成方法. 同样以酰胺和磺酰胺作为 原料, 加入二价锌作为催化剂, 重氮乙酸乙酯为添加物 可实现高通用性的 $N$-磺基脒合成. 反应的主要转化是 锌离子和重氮化合物形成金属卡宾，之后该卡宾对酰胺 进行活化，促进磺酰胺对酰胺羰基碳的加成. 脱去金属 后, 得到的 $N, O$-缩酰胺分解，再次脱去 $\alpha$-差基酯就可得 到目标产物(Scheme 19). 后续研究证实, 采用二价锰催 
化剂 $\mathrm{Mn}\left(\mathrm{ClO}_{4}\right)_{2} \cdot 6 \mathrm{H}_{2} \mathrm{O}$, 在重氮乙酸乙酯辅助下, 也可以 通过卡宾活化的方式实现这类合成反应 ${ }^{[38]}$.

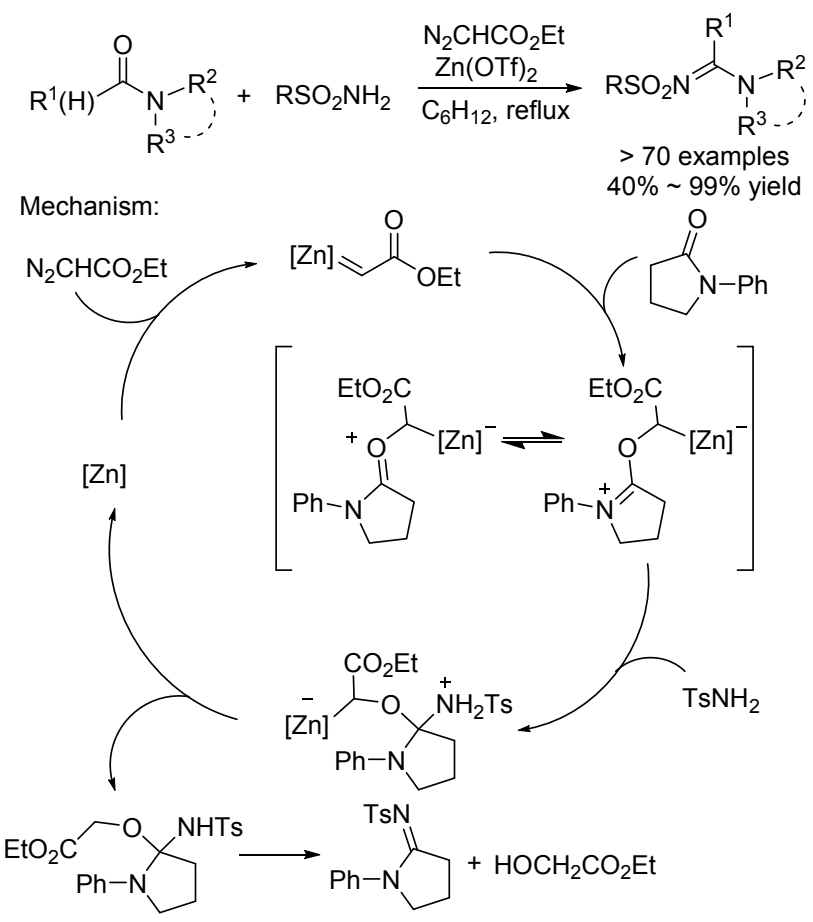

图式 19 金属卡宾促进磺酰胺和酰胺反应合成 $N$-磺基脒

Scheme 19 Metal carbene-promoted synthesis of $N$-sulfonyl amidines with amides and sulfonamides

张景萍小组 ${ }^{[39]}$ 运用 NBS 在 DMF 中介导芳基磺酰胺 与酰胺反应也实现了 $N$-磺基脒的合成. NBS 可能通过卤 素活化和氢键诱导的质子转移促进离子对中间体的生 成 ${ }^{[40]}$, 再经历半缩酰胺中间体的 $\mathrm{N}-\mathrm{Br}$ 键质子化以及脱 水就得到 $N$-磺基脒产物(Scheme 20).

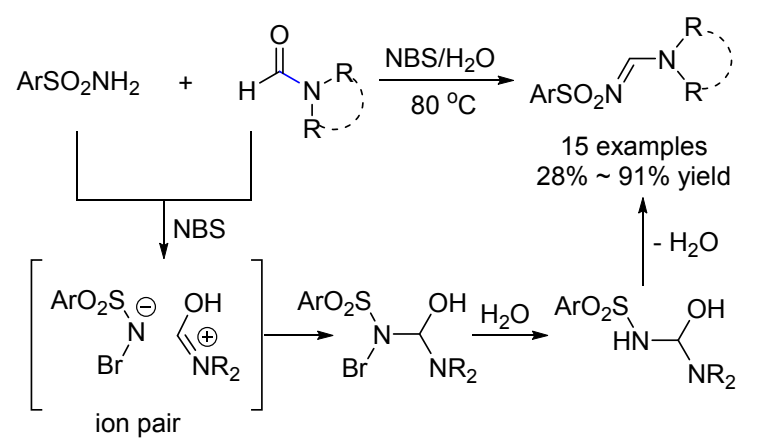

图式 20 NBS-水体系促进磺酰胺和酰胺反应合成 $N$-磺基脒 Scheme 20 NBS-water system-promoted synthesis of $N$-sulfonyl amidines with amides and sulfonamides

Odell 等 ${ }^{[41]}$ 以磺酰叠氮与酰胺为原料, 在低 $\mathrm{CO}$ 压 力下, 钯催化二者串联反应也生成 $N$-磺基脒. 反应须在 文献报道过的双室反应器 ${ }^{[42]}$ 中进行. 室一中加入底物 与钯催化剂, 室二中则加入六羰基钼 $\mathrm{Mo}(\mathrm{CO})_{6}$ 和 $\mathrm{DBU}$ 反应以提供 $\mathrm{CO}$. 反应的关键转化是磺酰叠氮在钯催化
下和羰基形成磺酰基异氰酸酯中间体。该中间体与酰胺 $[2+2]$ 环加成, 并脱羧形成目标产物(Scheme 21).

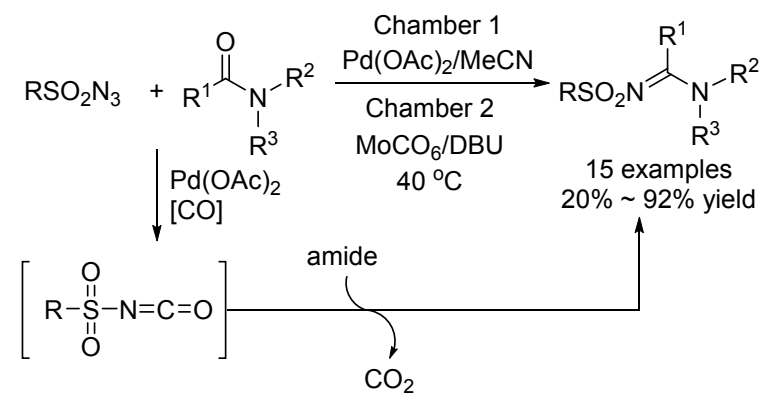

图式 21 钯催化磺酰叠氮和酰胺反应合成 $N$-磺基脒 Scheme 21 Palladium-catalyzed synthesis of $N$-sulfonyl amidines with amides and sulfonyl azides

Adolfsson 小组 ${ }^{[43]}$ 以 $\mathrm{Mo}(\mathrm{CO})_{6}$ 为羰基源, 在 1,1,3,3四甲基二硅氧烷(TMDS)辅助下, 以乙酸乙酯作溶剂, 在 $60{ }^{\circ} \mathrm{C}$ 也实现了类似通过磺酰叠氮和酰胺直接反应合 成 $N$-磺基脒的方法(Eq. 7)

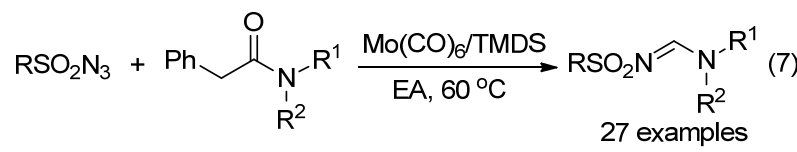

$$
\begin{aligned}
& 39 \% \sim 83 \% \text { yield }
\end{aligned}
$$

胡跃飞等 ${ }^{[44]}$ 以磺酰叠氮和酰胺作为反应物, 以炔 酮为辅助剂在 $\mathrm{CuI}$ 催化下实现了 $N$-磺基脒的合成. 该方 法采用铜催化的叠氮一炔环加成反应 $(\mathrm{CuAAC})$ 以及串联 放氮开环的经典转化得到烯酮亚胺中间体，并原位和酰 胺 $[2+2]$ 环加成得到四元环中间体后, 经过开环重组, 脱去炔片段就得到磺基榺产物，为酰胺参与的脒合成提 供了一种新方法(Scheme 22).

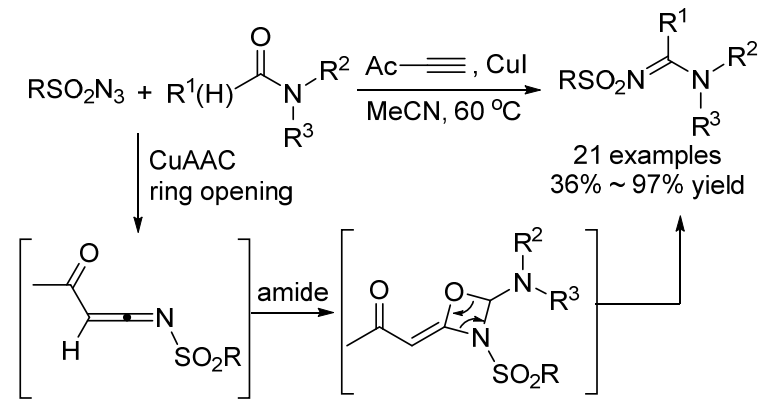

图式 22 端炔促进的磺酰叠氮和酰胺反应合成 $N$-磺基脒 Scheme 22 Terminal alkyne-promoted synthesis of $N$-sulfonyl amidines with amides and sulfonyl azides

\section{5 其它合成方法}

除了以上较为系统的合成方法，文献还报道了不少 其它类型的用于 $N$-磺基榺合成的反应模式. 例如 Hatanaka 小组 ${ }^{[45]}$ 报道了不需要任何催化剂或添加剂条 
件下硫代酰胺和磺酰叠氮反应合成 $N$-磺基脒的方法. 反应以乙醇或水为介质，同时适用于环状和非环状的硫 代酰胺. 反应可能的机理是硫代酰胺和叠氮经过偶极环 化反应生成硫代三唑啉中间体, 该环系不稳定, 通过开 环脱硫氮生成目标产物磺基脒(Scheme 23).

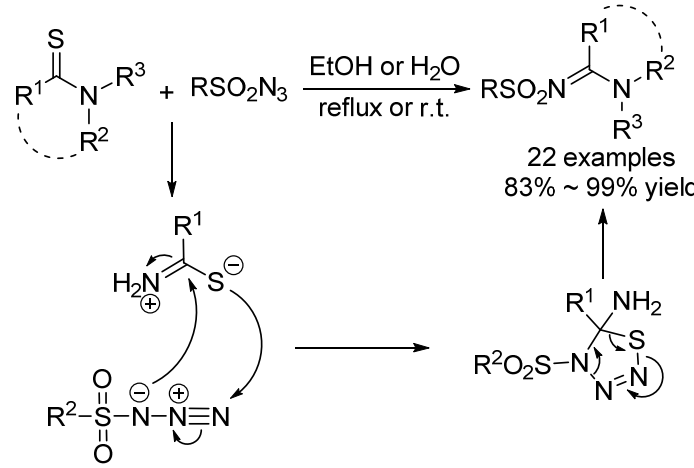

图式 23 磺酰叠氮和硫代酰胺反应合成 $N$-磺基脒

Scheme 23 Synthesis of $N$-sulfonyl amidines via the reactions of thioamides and sulfonyl azides

Hassan 等 ${ }^{[46]}$ 则报道了 $N$-磺基呋啶类结构和硫代酰 胺反应合成 $N$-磺基脒的方法. 在氯化锌催化下，吟啶环 被亲核硫位点进攻开环, 经历分子内氮亲核环化得到 $S, N$-五元环中间体, 该中间体经过开环脱去环硫乙烷得 到 $N$-磺基脒产物(Scheme 24).

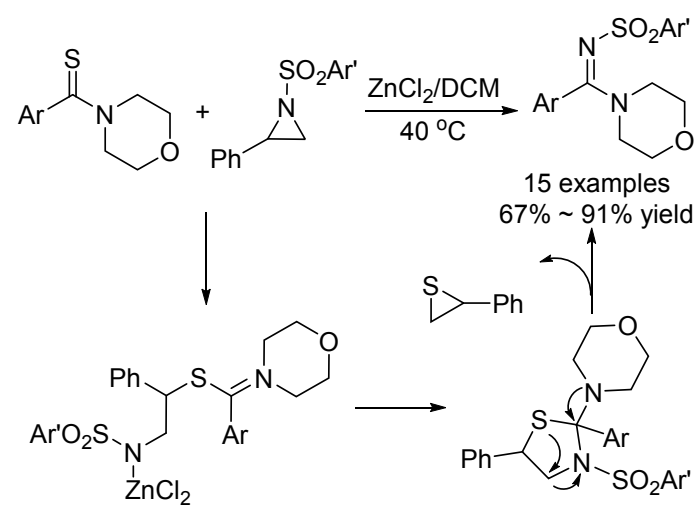

图式 $24 N$-磺基呋啶和硫代酰胺反应合成 $N$-磺基脒 Scheme 24 Synthesis of $N$-sulfonyl amidines via the reactions of thioamides and sulfonyl aziridines

Stahl 和 Kim 报道 ${ }^{[47]}$ 铜催化三组分氧化偶合合成 $N-$ 磺基脒的方法. 以 $\mathrm{Cu}(\mathrm{OTf})_{2}$ 为催化剂, 端炔、仲胺和磺 酰胺反应可得到 $N$-磺基脒(Eq. 8). 反应可能经历不同的 途径进行: 一是炔和磺胺先氧化偶联并异构化得到烯酤 亚胺, 再发生胺加成和异构化得到产物; 另一种可能途 径则是炔和胺酰经过氢胺化反应得到烯胺, 再氧化或异 构化得到磺基脒.

徐新芳小组 ${ }^{[48]}$ 报道了在 NaI/TBHP 催化体系中, 甲 基叔胺和磺酰胺反应合成 $N$-磺基咪唑的方法. 该方法 的主要原理是利用过氧化物单电子转移氧化模式, 甲基

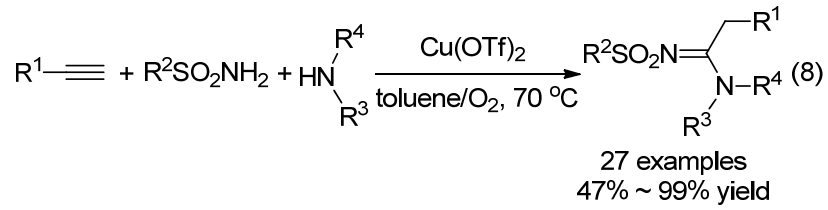

叔胺原位氧化成亚胺阳离子. 磺酰胺对亚胺离子加成再 进一步氧化就得到 $N$-磺基脒(Scheme 25).

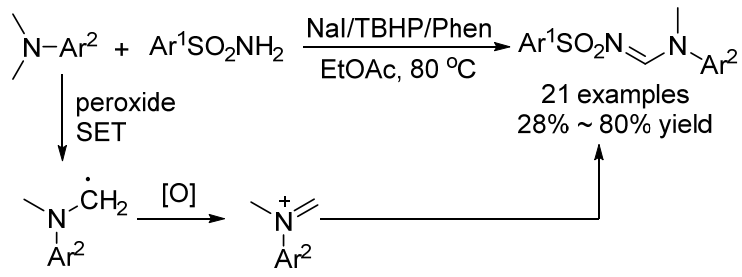

图式 25 甲基叔胺和磺酰胺反应合成 $N$-磺基脒 Scheme 25 Synthesis of $N$-sulfonyl amidines via the reactions of methyl tertiary amines and sulfonamides

此外, 易维银等 ${ }^{[49]}$ 以碘化亚铜为催化剂, 在碱性条 件和室温下于二氯甲烷水溶液中搅拌, 磺酰叠氮和氯化 肜反应合成了 $\mathrm{NH}_{2}$ 官能化 $N$-磺基脒. 反应采用端炔作 为辅助物促进反应. 控制实验表明在没有端炔或水的情 况下, 磺酰叠氮和氯化肜在标准条件下无法获得目标产 物. 该方法获得了氮原子上没有取代的 $N$-磺基脒(Eq. 9).

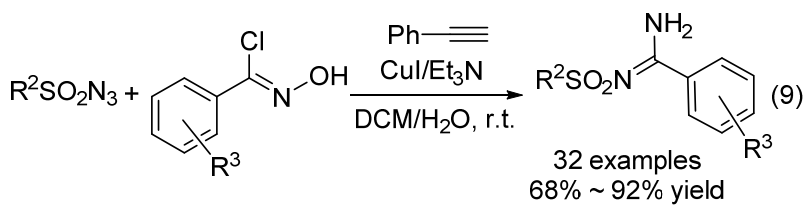

最近，毕锡和等 ${ }^{[50]}$ 通过银催化策略，采用端炔、三 甲基硅基叠氮、芳基亚磺酸钠和磺酰叠氮多组分反应合 成了 $N$-磺基脒. 反应经历了炔基碳-碳键的断裂，主要 转化包括已知的银催化下炔、三甲基硅基叠氮和亚磺酸 钠反应生成烯胺 ${ }^{[51]}$, 烯胺和磺酰叠氮环加成得到三唑 啉中间体，再经历典型的开环脱重氮转化得到 $N$-磺基 脒(Scheme 26).

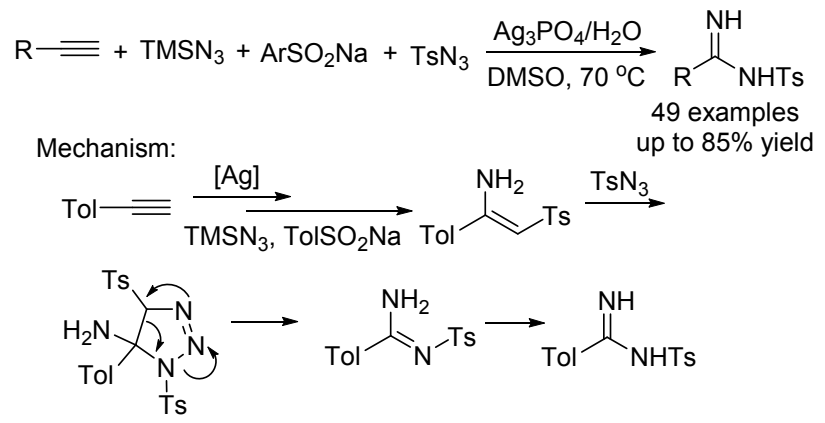

图式 26 银催化多组分反应合成 $N$-磺基脒

Scheme 26 Synthesis of $N$-sulfonyl amidines via Ag-catalyzed multicomponent reactions 
陈自立等 ${ }^{[52]}$ 以磺酰胺、芳基重氮盐和烷基腈为底 物, 在二氯乙烷中加热, 通过三组分反应的方式也可以 得到 $N$-磺基脒, 反应对无取代以及 $N$-取代的磺酰均兼 容, 且在放大反应时也取得了较好的结果(Eq. 10).

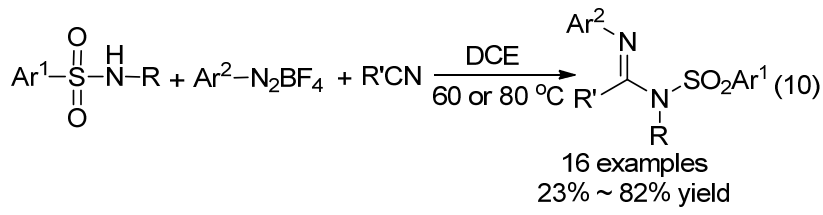

万小兵等 ${ }^{[53}$ 通过铜催化, 发展了两分子重氮乙酸 酯、磺酰胺以及腈衍生物的多组分反应合成 $N$-磺基脒的 方法. 反应在空气氛围下, $80{ }^{\circ} \mathrm{C}$ 加热条件下进行. 该体 系对底物有优秀的兼容性, 酯基和芳基腈都可以顺利参 与该反应, 得到相应的 $N$-磺基脒产物. 如 Scheme 27 所 示, 反应过程首先是叠氮和铜形成金属卡宾, 之后该中 间体和磺酰胺反应通过两种途径得到目标产物。一是该

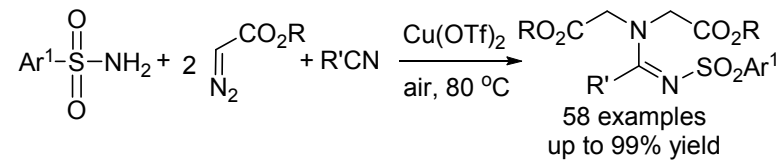

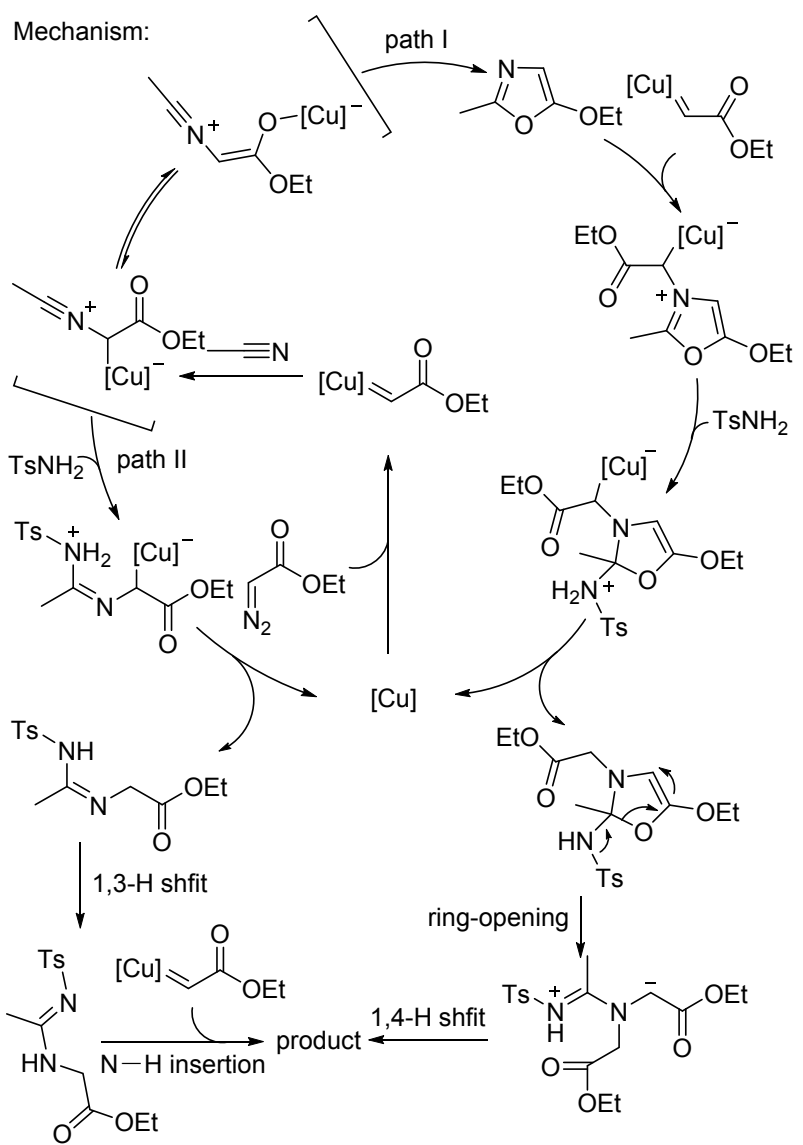

图式 27 铜催化磺酰胺和叠氮串联反应合成 $N$-磺基榺 Scheme 27 Synthesis of $N$-sulfonyl amidines via copper-catalyzed reactions of sulfonamides and diazo compounds
中间体直接分子内环化生成噁唑中间体之后再和铜卡 宾中间体经历系列转化后得到目标产物(path I); 另一可 能途径则是卡宾先和磺酰胺反应，得到 $\mathrm{NH}$-结构的磺基 醚，该中间体再次和叠氮酯发生 $\mathrm{N}-\mathrm{H}$ 和另一分子叠氮 酮的插入反应得到最终产物(path II).

\section{6 结论}

作为重要的有机原料以及多个功能有机分子中的 重要片段, $N$-磺基脒类化合物一直受到重点关注，而且 相关研究也取得了重要进展. 大量不同催化方法以及不 同类型的反应方法已经被发展并用于结构多样性的 $N$ 磺基脒类化合物的合成. 此外应当看到的是，基于已有 的方法而言， $N$-磺基脒的合成也还有很大的提升空间. 例如，许多合成方法都涉及分子片段断裂，在原子经济 方面还不完全理想. 此外，部分反应还存在使用过渡金 属催化、过氧化物氧化等较为剧烈的反应条件等问题. 因此，对于 $N$-磺基脒类化合物的合成，还有待更多的研 究和探索, 简单底物如胺、酰胺、磺酰胺等的直接转化、 温和条件下的反应模式以及兼容于 $N$-磺基脒之外其它 取代榺类化合物的合成方法等仍然是重要的研究课题.

\section{References}

[1] (a) Schade, D.; Kotthaus, J.; Riebling, L.; Kotthaus, J.; MüllerFielitz, H.; Raasch, W.; Koch, O.; Seidel, N.; Schmidtke, M.; Clement, B. J. Med. Chem. 2014, 57, 759.

(b) Liu, Y.; Hu, X.; Wu, Y.; Zhang, W.; Chen, X.; You, X.; Hu, L. Eur. J. Med. Chem. 2018, 150, 771.

(c) Shriner, R. L.; Neumann, F. W. Chem. Rev. 1944, 35, 351.

(d) Kumar, T.; Verma, D.; Menna-Barreto, R. F. S.; Valenca, W. O.; da Silva, E. N.; Namboothiri, I. N. N. Org. Biomol. Chem. 2015, 13, 1996.

(e) He, H.; Xia, Q.; He, H. Chin. J. Org. Chem. 2019, 39, 2295 (in Chinese).

(何海峰，夏芹，贺红武，有机化学， 2019, 39, 2295.)

(f) Wu, N.; Cheng, L.; Wang, J.; Yu, J.; Xing, J.; Xu, T.; Wei, Y. Chin. J. Org. Chem. 2019, 39, 852 (in Chinese).

(吴宁捷，程龙，王剑，郁季平，邢家华，许天明，魏优昌，有机 化学, 2019, 39, 852.)

[2] (a) Barker, J.; Kilner, M. Coord. Chem. Rev. 1994, 133, 219.

(b) Nguyen, T. L.; Panchal, R. G.; Topol, I. A.; Lane, D.; Kenny, T.; Burnett, J. C.; Hermone, A. R.; McGrath, C.; Burt, S. K.; Gussio, R.; Bavari, S. J. Mol. Struct. 2007, 821, 139.

(c) Yuan, J.; Li, J.; Wang, B.; Sun, S.; Cheng, J. Tetrahedron Lett. 2017, $58,4783$.

[3] (a) Katz, B. A.; Clark, J. M.; Finer-Moore, J. S.; Jenkins, T. E.; Johnson, C. R.; Ross, M. J.; Luong, C.; Moore, W. R.; Stroud, R. M. Nature 1998, 391, 608.

(b) Kotthaus, J.; Steinmetzer, T.; van de Locht, A.; Clement, B. J. Enzyme Inhib. Med. Chem. 2011, 26, 115.

(c) Mackman, R. L.; Katz, B. A.; Breitenbucher, J. G.; Hui, H. C.; Verner, E.; Luong, C.; Liu, L.; Sprengeler, P. A. J. Med. Chem. 2001, 44, 3856.

[4] Lee, M. Y.; Kim, M. H.; Kim, S. H.; Kim, B. T.; Jeong, I. H.; Chang, S.; Kim, H.; Chang, S.-Y. Bioorg. Med. Chem. Lett. 2010, 20,541 .

[5] Song, Z. L.; Chen, H.-L.; Wang, Y.-H.; Goto, M.; Gao, W.-J.; Cheng, P.-L.; Morris-Natschke, S. L.; Liu, Y.-Q.; Zhu, G.-X.; Wang, 
M.-J.; Lee, K.-H. Bioorg. Med. Chem. Lett. 2015, 25, 2690.

[6] Suja, T. D.; Divya, K. V. L.; Naik, L. V.; Ravi Kumar, A.; Kamal, A Bioorg. Med. Chem. Lett. 2016, 26, 2072.

[7] Luke, R. O.; Shiao, Y. C. J. Org. Chem. 2017, 82, 2515.

[8] (a) Ran, R.-Q.; Xiu, S.-D.; Li, C. Org. Lett. 2014, 16, 6394. (b) Nandi, G. C.; Jesin, I. Adv. Synth. Catal. 2018, 360, 2465.

(c) van Vliet, K. M.; Polak, L. H.; Siegler, M. A.; van der Vlugt, J. I.; Guerra, C. F.; de Bruin, B. J. Am. Chem. Soc. 2019, 141, 15240. (d) Aly, A. A.; Brase, S.; Gomaa, M. A.-M. ARKIVOC 2018, vi, 85.

[9] Xu, X.; Li, X.; Ma, L.; Ye, N.; Weng, B. J. Am. Chem. Soc. 2008, 130,14048

[10] Li, X.; Xu, X.; Ge, Z.; Cheng, D.; Ma, L.; Lu, C.; Zhang, Q.; Yao, N.; Li, X. Org. Lett. 2010, 12, 897.

[11] Liu, N.; Tang, B. Y.; Chen, Y.; He, L. Eur. J. Org. Chem. 2009, 2059.

[12] Bi, W.-Z.; Zhang, W.-J.; Li, Z.-J.; Xia, X.-Y.; Chen, X.-L.; Qu, L.-B.; Zhao, Y.-F. Eur. J. Org. Chem. 2019, 6071

[13] Wang, S.; Wang, Z.; Zheng, X. Chem. Commun. 2009, 7372.

[14] (a) Rouzi, A.; Hudabaierdi, R.; Wusiman, A. Tetrahedron 2018, 74, 2475.

(b) Jian, Y.; Chen, M.; Yang, C.; Xia, W. Synthesis 2019, 51, 4425.

[15] Shojaei, S.; Ghasemi Z.; Shahrisa, A. Tetrahedron Lett. 2017, 58, 3957.

[16] Gui, J.; Xie, H.; Jiang, H.; Zeng, W. Org. Lett. 2019, 21, 2804.

[17] Ding, R.; Chen, H.; Xu, Y. L.; Tang, H. T.; Chen, Y. Y.; Pan, Y. M. Adv. Synth. Catal. 2019, 361, 3656.

[18] Wang, Z.; Zhang, L. Wang, S.; Wan, C.; Zha, Z.; Du, J.; Wang, Z. Chem. Commun. 2011, 47, 5488.

[19] Chen, J.; Guo, Y.-P.; Sun, M.-H.; Fan, G.-T.; Zhou, L. Chem. Commun. 2014, 50, 12367.

[20] Fusco, R.; Bianchetti, G.; Pocar, D.; Ugo, R. Chem. Ber. 1963, 96, 802.

[21] Gao, T.; Zhao, M.; Meng, X.; Li, C.; Chen, B. Synlett 2011, 1281.

[22] Efimov, I.; Beliaev, N.; Beryozkina, T.; Slepukhin, P.; Bakulev, V. Tetrahedron Lett. 2016, 57, 1949.

[23] Zheng, X.; Wan, J.-P. Adv. Synth. Catal. 2019, 361, 5690.

[24] Wang, G.; Guo, Y.; Wan, J.-P. Chin. J. Org. Chem. 2020, 40, 645 (in Chinese). (王国栋，郭艳辉，万结平，有机化学, 2020, 40, 645.)

[25] Bae, I.; Han, H.; Chang, S. J. Am. Chem. Soc. 2005, 127, 2038.

[26] Chang, S.; Lee, M.; Jung, D. Y.; Yoo, E. J.; Cho, S. H.; Han, S. K. J. Am. Chem. Soc. 2006, 128,12366

[27] Kim, J.; Lee, S. Y.; Lee, J.; Do, Y.; Chang, S. J. Org. Chem. 2008, $73,9454$.

[28] Zhou, Y.; Webber, S. E.; Murphy, D. E.; Li, L.-S.; Dragovich, P. S.; Tran, C. V.; Sun, Z.; Ruebsam, F.; Shah, A. M.; Tsan, M.; Showalter, R. E.; Patel, R.; Li, B.; Zhao, Q.; Han, Q.; Hermann, T.; Kissinger, C. R.; LeBrun, L.; Sergeeva, M. V.; Kirkovsky, L.
Bioorg. Med. Chem. Lett. 2008, 18, 1413.

[29] Murugavel, G.; Punniyamurthy, T. J. Org. Chem. 2015, 80, 6291.

[30] Jagadale, M.; Bhange, P.; Salunkhe, R.; Bhange, D.; Rajmane, M.; Rashinkar, G. Appl. Catal. A: Gen. 2016, 511, 95

[31] Kim, M. J.; Kim, B. R.; Lee, C. Y.; Kim, J. Tetrahedron Lett. 2016, 57, 4070 .

[32] Yavari, I.; Ahmadian, S.; Majid, G.-D.; Solgi, Y. Tetrahedron Lett. 2011, 52, 668

[33] He, X. W.; Shang, Y.; Hu, J.; Ju, K.; Jiang, W.; Wang, S. Sci. China, Chem. 2012, 55, 214

[34] Yao, B.; Shen, C.; Liang, Z.; Zhang, Y. J. Org. Chem. 2014, 79, 936.

[35] (a) Pettit, G. R.; Kadunce, R. E. J. Org. Chem. 1962, 27, 4566.

(b) Gazvoda, M.; Kočevar, M.; Polance, S. Eur. J. Org. Chem. 2013, 5381.

(c) Chandna, N.; Chandak, N.; Kumar, P.; Kapoor, J. K.; Sharma, P. K. Green Chem. 2013, 15, 2294.

(d) Jeong, Y.; Ban, J.; Lim, M.; Rhee, H. Synthesis 2018, 50, 1867.

[36] Wan, X.; Chen, S.; Xu, Y. Org. Lett. 2011, 13, 6152

[37] Chen, J.; Long, W.; Fang, S.; Yang, Y.; Wan, X. Chem. Commun. 2017, 53, 13256 .

[38] Chen, J.; Long, W.; Yang, Y.; Wan, X. Org. Lett. 2018, 20, 2663.

[39] Niu, Z.; Lin, S.; Dong, Z.; Sun, H.; Liang, F.; Zhang, J. Org. Biomol. Chem. 2013, 11, 2460

[40] Wei, Y.; Lin, S.; Liang, F. Org. Lett. 2012, 14, 4202.

[41] Chow, S. Y.; Odell, L. R. J. Org. Chem. 2017, 82, 2515.

[42] Friis, S. D.; Lindhardt, A. T.; Skrydstrup, T. Acc. Chem. Res. 2016, 49, 594.

[43] Trillo, P.; Slagbrand, T.; Tinnis, F.; Adolfsson, H. ChemistryOpen 2017, 6, 484

[44] Yang, W.; Huang, D.; Zeng, X.; Luo, D.; Wang, X.; Hu, Y. Chem. Commun. 2018, 54, 8222.

[45] Aswad, M.; Chiba, J.; Tomohiro, T.; Hatanaka, Y. Chem. Commun. 2013, 49, 10242 .

[46] Khadijeh, H.; Hassan, Z.-B. Synlett 2014, 25, 2044

[47] Kim, J.; Stahl, S. S. J. Org. Chem. 2015, 80, 2448.

[48] Zheng, Y.; Mao, J. C.; Chen, J.; Rong, G. W.; Liu, D. F.; Yan, H.; Chi, Y. J.; Xu, X. F. RSC Adv. 2015, 5, 50113.

[49] Yi, F.; Sun, Q.; Sun, J.; Fu, C.; Yi, W. J. Org. Chem. 2019, 84, 6780.

[50] Liu, B.; Ning, Y.; Virelli, M.; Zanoni, G..; Dnderson, E. A.; Bi, X. J. Am. Chem. Soc. 2019, 141, 1593.

[51] Ning, Y.; Ji, Q.; Liao, P.; Anderson, E. A.; Bi, X. Angew. Chem., Int. Ed. 2017, 56, 13805

[52] Zhang, Y.; Chen, Z. Tetrahedron Lett. 2018, 59, 4183.

[53] Chen, J.; Long, W.; Zhao, Y.; Li, H.; Zheng, Y.; Lian, P.; Wan, X. Chin. J. Chem. 2018, 36, 857.

(Cheng, F.) 